\title{
COMPOSTOS LAMELARES COM POTENCIAL ATIVIDADE CATALÍTICA NA SÍNTESE DE BIODIESEL POR MEIO DE TRANSESTERIFICAÇÃO
}

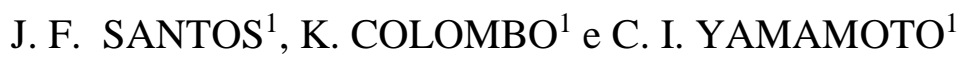 \\ ${ }^{1}$ Universidade Federal do Paraná, Departamento de Engenharia Química \\ E-mail para contato: jessica1209a@gmail.com
}

\begin{abstract}
RESUMO - Catalisadores atuando em meio heterogêneo facilitam o processo de separação dos sólidos e dos produtos de reação, permitindo o seu reuso e/ou reciclagem. As matrizes mais utilizadas para a intercalação de espécies aniônicas com atividade catalítica são os hidróxidos duplos lamelares (HDL), os hidroxissais lamelares (HSL) e os hidroxissais duplos lamelares (HSDL), possuindo respectivamente as formulações genéricas: $\mathrm{M}^{+2}{ }_{1-\mathrm{x}} \mathrm{M}^{+3}{ }_{\mathrm{x}}(\mathrm{OH})_{2}\left(\mathrm{~A}^{-}\right.$ $\left.{ }^{n}\right)_{\mathrm{x} / \mathrm{n}} \cdot \mathrm{yH}_{2} \mathrm{O}, \mathrm{M}^{+2}(\mathrm{OH})_{2-\mathrm{x}}\left(\mathrm{A}^{-\mathrm{x}}\right)_{\mathrm{x} / \mathrm{n}} \cdot \mathrm{yH}_{2} \mathrm{O}$ e $\mathrm{M}_{\mathrm{a}}{ }^{+2}{ }_{1-\mathrm{y}} \mathrm{M}_{\mathrm{b}}{ }^{+2} \mathrm{y}(\mathrm{OH})_{2-\mathrm{x}}\left(\mathrm{A}^{-\mathrm{x}}\right)_{\mathrm{x} / \mathrm{n}} \cdot \mathrm{yH}_{2} \mathrm{O}$ onde onde $\mathrm{M}^{+2}$ e $\mathrm{M}^{+3}$ são metais divalentes e trivalentes, $\mathrm{A}^{-\mathrm{x}}$, o ânion intercalado e $\mathrm{M}_{\mathrm{a}}^{+2}$ e $\mathrm{M}_{\mathrm{b}}{ }^{+2}$ representam os dois metais da estrutura. $\mathrm{O}$ presente trabalho teve como objetivo sintetizar e caracterizar o HSDL de $\mathrm{Zn} / \mathrm{Ni}$. O material foi caracterizado por difração de raios $X$ (DRX) e espectroscopia vibracional na região do infravermelho com transformada de Fourier (FTIR). Após caracterização, o hidroaxicetato de zinco/níquel foi testado como catalisador para a reação de transesterificação metílica de óleo de soja, apresentando elevada atividade catalítica. Além de ser facilmente separada do meio reacional e manter sua estrutura após vários ciclos de reação.
\end{abstract}

\section{INTRODUÇÃO}

O biodiesel se apresenta como fonte alternativa em relação às fontes esgotáveis de combustíveis fósseis. Tendo em vista que além de renovável é menos poluente, pois apresenta um baixo perfil de emissões de monóxido de carbono e material particulado e não libera compostos de enxofre. O biodiesel também pode ser lubrificante na forma de líquido biodegradável, o qual pode ser misturado ao diesel, ou mesmo substitui-lo em uma gama de aplicações como caminhões a diesel, geradores de eletricidade, equipamentos para mineração, motores marítimos e frotas de ônibus para transporte público (Nandiwale e Bokade, 2014; Cordeiro et al., 2011; Santos, 2016).

Usualmente o biodiesel é produzido por catálise em meio homogêneo, pelo uso de hidróxido de sódio ou hidróxido de potássio. Todavia, a transesterificação via catálise homogênea gera grandes desperdícios, oriundos, principalmente das etapas de lavagem para separação dos produtos obtidos. O que pode ser minimizado pela utilização de catalisadores em meio heterogêneo, que apresentam vantagens técnicas e ambientais, representadas pela facilidade das operações posteriores à reação principal (etapas de purificação), pela 
possibilidade de reuso do catalisador ao longo de sua vida útil e por gerar menor quantidade de efluentes (Coelho et al, 2017; Cordeiro et al.,2011).

Uma ampla gama de sólidos tem sido proposta como potenciais catalisadores na síntese de biodiesel, tais como os óxidos de metais alcalino-terrosos, hidróxidos ou sais suportados em alumina, zeólitas, compostos de coordenação, líquidos iônicos e materiais lamelares, os quais incluem os hidróxidos duplos lamelares (HDL) e os HSL. A atividade catalítica está relacionada a natureza ácida ou básica dos sítios ativos que esses sólidos possuem (Cordeiro et al., 2011).

No presente trabalho será analisado o HSDL hidroxiacetato de zinco-níquel como potencial catalisador para produção de biodiesel, o qual foi sintetizado e caracterizado.

\subsection{Objetivos}

- Sintetizar o hidroxiacetato de Zinco-Níquel por reação Sal e Óxido;

- Realizar as caracterizações do composto através das seguintes técnicas: Difração de Raios X (DRX), Espectroscopia vibracional na região do Infravermelho com Transformada de Fourier (FTIR).

- Realizar testes preliminares de atividade catalítica do composto para reações de transesterificação em meio heterogêneo para produção de biodiesel;

- Quantificar a porcentagem de éster nos produtos reacionais obtidos através de cromatografia em fase gasosa;

\section{REVISÃO BIBLIOGRÁFICA}

O biodiesel é obtido pela transesterificação de óleos vegetais ou de gorduras animais com álcoois de cadeia curta. Esse processo consiste na transformação de um éster carboxílico em outro, por meio da troca do grupo RO- presente no éster original, por outro grupo semelhante proveniente de um álcool, na presença de um catalisador ou não, (condições supercríticas), para produzir a glicerina e mono-alquil ésteres (biodiesel) (Boey et al., 2011).

Atualmente para a produção de biodiesel, a partir de óleos vegetais/gordura utilizam-se $\mathrm{KOH}$ ou $\mathrm{NaOH}$ como catalisador em meio homogêneo. A vantagem deste processo se deve à elevada conversão de triacilglicerol em ésteres metílicos/etílicos, condições brandas de reação e curto tempo de reação (Kouzu et al, 2008).

Convencionalmente reações de esterificação são performadas via catálise em meio homogêneo. No entanto, a utilização de catalisadores homogêneos encontra severas limitações, dessa forma, como resposta ao desafio tecnológico da produção sustentável de biodiesel está o desenvolvimento de catalisadores em meio heterogêneo. Esse tipo de catálise promove uma mais fácil separação dos produtos, baixo perfil de emissões poluentes e não coloca em risco de corrosão o equipamento (Nandiwale e Bokade, 2014).

Os compostos lamelares incluem uma série de materiais, como os hidroxissais lamelares (HSL), os carboxilatos lamelares, os hidróxidos duplos lamelares e os óxidos estruturados oriundos da calcinação controlada de HDL. Todos são compostos pelo empilhamento de unidades chamadas de lamelas, as quais se mantêm unidas por forças de baixa intensidade do tipo Van der Waals, interações eletrostáticas e ligações de hidrogênio (Marangoni, 2009; Cordeiro et al., 2011).

Os HSL possuem uma fórmula genérica $\mathrm{M}^{+2}(\mathrm{OH})_{2-\mathrm{x}}\left(\mathrm{A}^{-\mathrm{x}}\right)_{\mathrm{x} / \mathrm{n}} \cdot \mathrm{yH}_{2} \mathrm{O}$ onde $\mathrm{M}^{+2}$ representa um metal divalente como o $\mathrm{Mg}^{2+}, \mathrm{Ni}^{2+}, \mathrm{Zn}^{2+}, \mathrm{Ca}^{2+}, \mathrm{Cd}^{2+}, \mathrm{Co}^{2+}$ ou $\mathrm{Cu}^{2+}$ e $\mathrm{A}^{-\mathrm{x}}$, um ânion que 
substitui parcialmente o íons hidroxila estruturais (Arizaga et al., 2007; Kandare e Hossenlopp, 2006). Os ânions variam, sendo os mais comuns o $\mathrm{Cl}^{-}, \mathrm{NO}_{3}^{-}, \mathrm{CO}_{3}^{-2}$, acetato e carboxilatos de cadeias mais longas como aqueles derivados de ácidos graxos.

Os HSDL são uma classe derivada dos HSL em que dois ou até três metais ocupam sítios específicos nas lamelas do material lamelar, fórmulas podem ser descritas como: $\mathrm{M}_{\mathrm{a}}{ }^{+2}$ ${ }_{\mathrm{y}} \mathrm{M}_{\mathrm{b}}{ }^{+2} \mathrm{y}(\mathrm{OH})_{2-\mathrm{x}}\left(\mathrm{A}^{-\mathrm{x}}\right)_{\mathrm{x} / \mathrm{n} .} \mathrm{yH} \mathrm{H}_{2} \mathrm{O}$ onde $\mathrm{M}_{\mathrm{a}}{ }^{+2}$ e $\mathrm{M}_{\mathrm{b}}{ }^{+2}$ representam os dois metais da estrutura do HSDL (Arizaga et al., 2007; Yamanaka et al., 2006).

Ainda não existem muitos estudos sobre a potencial atividade catalítica dos HSDL na transesterificação metílica de óleo de soja.

\section{EXPERIMENTAL}

\subsection{Síntese do hidroxiacetato de $\mathrm{Zn} / \mathrm{Ni}$}

Para a síntese do hidroxiacetato de $\mathrm{Zn} / \mathrm{Ni}$ (HAZN) foram preparados $200 \mathrm{~mL}$ de uma solução de $\mathrm{Ni}\left(\mathrm{CH}_{3} \mathrm{COO}\right)_{2}$ (VETEC Quimica) $0,5 \mathrm{~mol} / \mathrm{L}$, à qual foram adicionados $4,07 \mathrm{~g}$ de $\mathrm{ZnO}$ (Synth). A reação foi mantida à $65^{\circ} \mathrm{C}$ sob agitação magnética $(750 \mathrm{rpm})$ durante 7 dias. Após este período, o material foi separado por centrifugação (4.000 rpm e 4 minutos) e lavado com água destilada sucessivas vezes (4 repetições). O sólido obtido foi então colocado em estufa à $65^{\circ} \mathrm{C}$ e deixado secar por 48 horas.

\subsection{Caracterização dos materiais}

As medidas de difração de raios X (XRD) dos precursores foram obtidas depositando-se os materiais em portas-amostra de vidro ou alumínio, utilizando-se um difratômetro Shimadzu modelo XRD-6000. Utilizou-se uma fonte de radiação da lâmpada de cobre $\mathrm{CuK} \alpha=1,5418 \AA$, corrente de $30 \mathrm{~mA}$ e tensão de $40 \mathrm{KV}$. Os difratogramas foram registrados com velocidade $2^{\circ}$. min- 1 e passo de 0,02 graus.

Os espectros de infravermelho (FTIR) foram obtidos pelo modo transmissão em um equipamento Bio-Rad, Modelo FTS 3500GX, mediante o uso de pastilhas de $\mathrm{KBr}$, com acumulação de 32 varreduras na faixa dos $400 \operatorname{aos} 4000 \mathrm{~cm}^{-1} \mathrm{e}$ resolução de $4 \mathrm{~cm}$.

Os ensaios de transesterificação metílica foram realizados com o óleo de soja refinado e metanol anidro, utilizando um reator modelo miniclave-drive da marca Buchiglassuster, constituído de um recipiente de aço com capacidade para $100 \mathrm{~mL}$, acoplado a um sistema de controle de aquecimento modelo Julabo®-HE4. Os experimentos foram conduzidos utilizando um planejamento fatorial total de 3 variáveis e 2 níveis com 3 experimentos no ponto central. De forma a variar a massa de catalisador, a temperatura de reação e a razão molar óleo/metanol. As condições operacionais fixas são: tempo de reação de $3 \mathrm{~h}$, e massa do óleo de $6 \mathrm{~g}$. O teor de ésteres foi analisado em um cromatógrafo à gás da marca SHIMADZU GC-210 com injetor AOC-5000 com detector FID e coluna capilar SUPELCOWAX $30 \mathrm{~m} \mathrm{x}$ $0,25 \mathrm{~mm} \times 0,25 \mu \mathrm{m}$. As medidas foram realizadas segundo a norma EN14103.

\section{RESULTADOS E DISCUSSÕES}

A tabela 1 mostra os resultados das conversões do óleo para éster. Observa-se que o maior valor de conversão térmica em éster foi de $6,4 \%$ a $140^{\circ} \mathrm{C}$ com razão molar $45: 1$, e o menor valor foi de $4,9 \%$ a $120^{\circ} \mathrm{C}$ e razão de $15: 1$. 
Observa-se também que todos os resultados (descontados das suas respectivas conversões térmicas) foram positivos, o que indica uma contribuição efetiva do HAZN na conversão do óleo de soja em éster.

As melhores conversões em éster $(88,80,79,60$ e 78,20\%) foram observadas à temperatura de $140{ }^{\circ} \mathrm{C}$, com diferenças significativas nos valores empregados de $\mathrm{RM}$ e porcentagem de catalisador, ao passo que os menores valores de conversão foram observados à temperatura de $120{ }^{\circ} \mathrm{C}, \mathrm{RM} 15: 1$ e $5 \%$ de catalisador $(25,9 \%)$ e no ponto central $(49,3 \mathrm{e}$ $48,8 \%)$.

A partir dos resultados da Tabela 1 é possível estabelecer os fatores de influência positiva ou negativa, em pontos percentuais, para cada variável ou interação entre eles. A análise dos efeitos isolados, bem como para as interações combinadas entre cada variável (Tabela 2) fornece dados importantes sobre a magnitude de cada termo. Dentre todos os termos, a razão molar foi o fator mais relevante (12\%). Tal fato é confirmado ao observar as reações 1 e 3, por exemplo: mantendo os valores RM e porcentagem de catalisador constantes, mas aumentando a RM de 15 para 45, a taxa de conversão $25,90 \%$ aumenta para $61,80 \%$. A mesma tendência é observada nas reações 6 e 8 , onde o mesmo tipo de mudança na RM leva a uma mudança na conversão de 78,20 para 88,80 , assim como no par reacional 5 e 7. A única exceção é observada no par reacional 2 e 4, em que o aumento da RM levou a uma redução na conversão.

Para a temperatura também foi constatado o seu efeito positivo, mas com uma magnitude um pouco menor em relação a RM, nas reações de esterificação $(11,25 \%)$. Tal fato é confirmado ao observar as reações 1 e 2, por exemplo: sob condições constantes de RM e porcentagem de catalisador, mas aumentando a temperatura de 120 para $140{ }^{\circ} \mathrm{C}$, a taxa de conversão $25,90 \%$ sobe para 79,60\%. A mesma tendência é observada nas reações 5 e 6 , onde o mesmo tipo de mudança na temperatura leva a uma mudança na conversão de 49,70 para 78,20 , assim como no par reacional 7 e 8 . A única exceção é observada no par reacional 3 e 4 , em que o aumento da temperatura levou a uma queda na conversão.

Dentre todos os efeitos isolados, o catalisador $(8,5 \%)$ apresentou a menor relevância. Tal fato pode ser constatado ao se observar que na maioria dos casos o aumento da RM levou a ligeiros aumentos nos valores de conversão nos pares reacionais $1 / 5,2 / 6$ e 3/7, com exceção do par 4/8, em que o acréscimo na conversão foi bem mais intenso.

A interação temperatura: catalisador apresentou efeito ligeiramente positivo no sistema $(1,75 \%)$, sendo que as interações temperatura: razão molar $(-0,75 \%)$ e razão molar: catalisador $(-5,50 \%)$ tiveram efeitos negativos para reações de transesterificação.

A fim de avaliar quais são os termos realmente insignificantes para o sistema, os resultados foram submetidos à análise de variância. Os valores de $\mathrm{p}$ inferiores a 0,05 são considerados significativos para os seus respectivos termos (CHEN et al., 2015).

Tabela 1 - Resultados da Transesterificação obtidas com o planejamento fatorial total aplicado com o HAZN como catalisador

\begin{tabular}{ccccccc}
\hline EXP & Temperatura $\left({ }^{\circ} \mathbf{C}\right)$ & $\begin{array}{c}\text { Razão } \\
\text { Molar }\end{array}$ & $\begin{array}{c}\text { Cat. } \\
(\boldsymbol{\%})\end{array}$ & $\begin{array}{c}\text { Conversão } \\
\text { Catalítica (\%) }\end{array}$ & $\begin{array}{c}\text { Conversão } \\
\text { Térmica (\%) }\end{array}$ & $\begin{array}{c}\text { Ganho de } \\
\text { Conversão (\%)* }\end{array}$ \\
\hline $\mathbf{1}$ & 120 & 15 & 5 & 25,9 & 4,9 & 21 \\
$\mathbf{2}$ & 140 & 15 & 5 & 79,6 & 5,4 & 74,2 \\
$\mathbf{3}$ & 120 & 45 & 5 & 61,8 & 6,2 & 55,6 \\
$\mathbf{4}$ & 140 & 45 & 5 & 46,1 & 6,4 & 39,7 \\
$\mathbf{5}$ & 120 & 15 & 10 & 49,7 & 4,9 & 44,8 \\
$\mathbf{6}$ & 140 & 15 & 10 & 78,2 & 5,4 & 72,8 \\
\hline
\end{tabular}




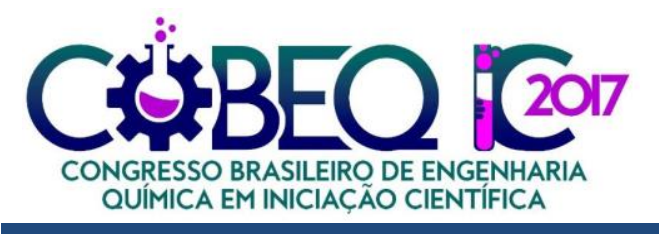

XII Congresso Brasileiro de Engenharia Química
em Iniciação Científica
UFSCar - São Carlos - SP
16 a 19 de Julho de 2017

\begin{tabular}{ccccccc}
\hline $\mathbf{7}$ & 120 & 45 & 10 & 64,5 & 6,2 & 58,3 \\
$\mathbf{8}$ & 140 & 45 & 10 & 88,8 & 6,4 & 82,4 \\
$\mathbf{9}$ & 130 & 30 & 7,5 & 49,3 & 5,2 & 44,1 \\
$\mathbf{1 0}$ & 130 & 30 & 7,5 & 48,8 & 5,2 & 43,6 \\
$\mathbf{1 1}$ & 130 & 30 & 7,5 & 53,4 & 5,2 & 48,2 \\
\hline
\end{tabular}

* Ganho de conversão $=($ Conversão catalítica $)-($ Conversão térmica + rampa de aquecimento)

Tabela 2 - Efeitos das variáveis isoladas e suas interações

\begin{tabular}{cc}
\hline Variável / Interação & Efeito (\%) \\
\hline Temperatura & 11,25 \\
Razão Molar & 12 \\
Catalisador & 8,5 \\
Temperatura: Razão Molar & $-0,75$ \\
Temperatura: Catalisador & 1,75 \\
Razão Molar: Catalisador & $-5,5$ \\
\hline
\end{tabular}

Tabela 3- Análise de variância

\begin{tabular}{c|ccc}
\hline Termos & Soma dos quadrados & F-valor & $\boldsymbol{p}$-valor \\
\hline Modelo & 3013,5 & 134,93 & 0,0010 \\
A: Temperatura & 1012,5 & 272,01 & 0,0005 \\
B: Razão Molar & 1152,0 & 309,49 & 0,0004 \\
C: \% catalisador & 578,0 & 155,28 & 0,0011 \\
AB & 4,5 & 1,21 & 0,3519 \\
AC & 24,5 & 6,58 & 0,0828 \\
BC & 242,0 & 65,01 & 0,004 \\
Falta de ajuste & 0,5 & 0,094 & 0,7884 \\
Erro puro & 10,67 & &
\end{tabular}

A análise de variância (Tabela 3) revelou que os termos A (temperatura), B (razão molar), C (catalisador) e a combinação entre razão molar e catalisador são parâmetros significativos no processo. A análise de regressão múltipla foi empregada nos dados, e o modelo de interação de dois fatores (2FI) foi escolhido como o mais adequado. A equação final do modelo (equação 1), de média 58,82 e desvio padrão de 1,93, pode ser observada abaixo:

conversão catalisada $=-118+0,75 \times \mathrm{T}+2,55 \times \mathrm{RM}-1,3 \times \mathrm{Cat}-0,005 \times \mathrm{T} * \mathrm{RM}+0,07$ $\times \mathrm{T}^{*}$ Cat $-0,1467 \times \mathrm{RM}^{*}$ Cat

$\left(\mathrm{T}=\right.$ temperatura $\left({ }^{\circ} \mathrm{C}\right) ; \mathrm{RM}=$ razão molar (álcool:óleo); cat $=$ catalisador $(\%)$ ).

$\mathrm{O}$ valor de $\mathrm{R}^{2}$ obtido para a resposta $(0,9963)$ foi maior do que 0,90 , o que indica a boa qualidade do modelo obtido. O valor adj- $\mathrm{R}^{2}$ obtido foi de 0,9889 para a resposta. $\mathrm{O}$ coeficiente de variação obtidos neste trabalho foi de 3,28. A Figura 1 mostra o gráfico de superfície de resposta para a temperatura, razão molar e conversão catalítica de acordo com a massa de catalisador utilizada. O ponto ideal para o modelo foi obtido por seleção de alguns parâmetros de conveniência, como menores quantidade de reagentes, mas priorizando um alto valor de conversão. Portanto, o melhor ponto sugerido para a conversão catalisada foi: 127,62 ${ }^{\circ} \mathrm{C}, 45: 1$ de razão molar e 5,41\% de catalisador, com uma conversão prevista de $69,3 \%$. 
Figura 1- Superfície de resposta com 5,41\% de catalisador

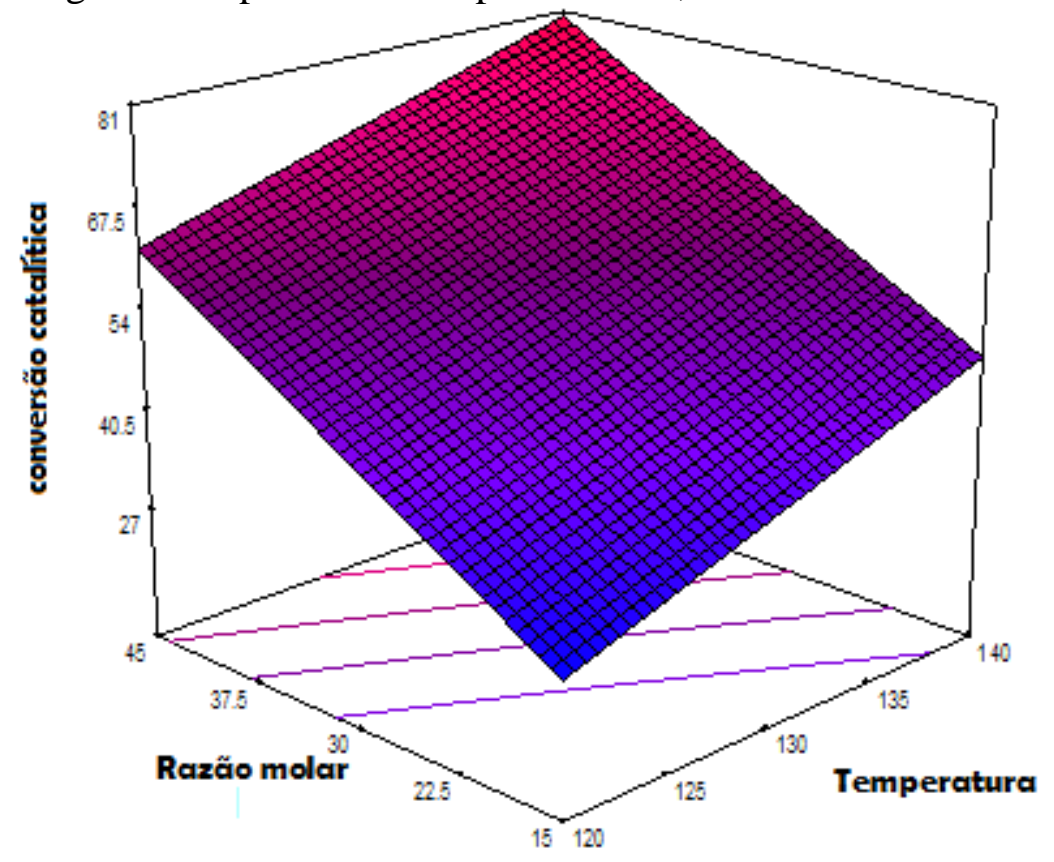

O HAZN também foi investigado por difração de raios $\mathrm{X}$ após um uso e dois reusos como catalisador na reação de transesterificação de óleo de soja com metanol (Figura 2).

Figura 2 - Difratogramas de raios X do material HAZN (a), após reação de transesterificação de óleo de soja com metanol (b), primeiro reuso (c), segundo reuso (d)

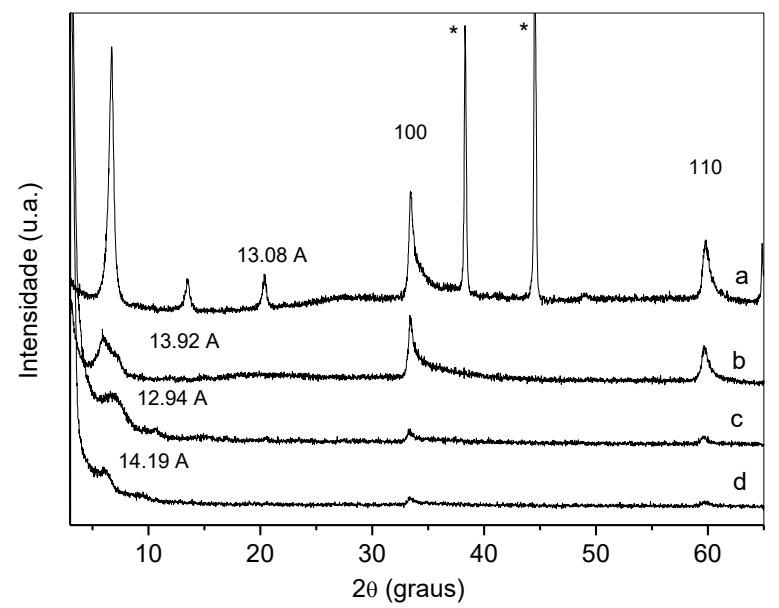

A distância basal calculada pelo pico de maior ordem possível para o HAZN $(n=3)$ foi 13,08 , e para os compostos (b), (c) e (d), utilizou-se o primeiro pico para o cálculo das distâncias basais, sendo que os respectivos valores foram obtidos: 13,92 ̊, 12,94 ̊ e 14,19 Å. Isso evidencia que após a utilização em um ciclo reacional na transesterificação de óleo de soja com metanol, o HAZN é recuperado, porém sua cristalinidade diminui, tendendo a amorficidade. Uma evidência sobre a preservação parcial da lamela do material é a 
manutenção dos picos de difração não basais, indexados como 100 e 110, em todos os difratogramas de raios $\mathrm{X}$.

O HAZN foi investigado por espectroscopia vibracional na região do infravermelho com transformada de Fourier após o uso e dois reusos como catalisador na reação de transesterificação de óleo de soja com metanol (Figura 3).

Figura 3 - Espectros de FTIR do composto HAZN (a), após reação de transesterificação de óleo de soja com metanol (b), primeiro reuso (c), segundo reuso (d).

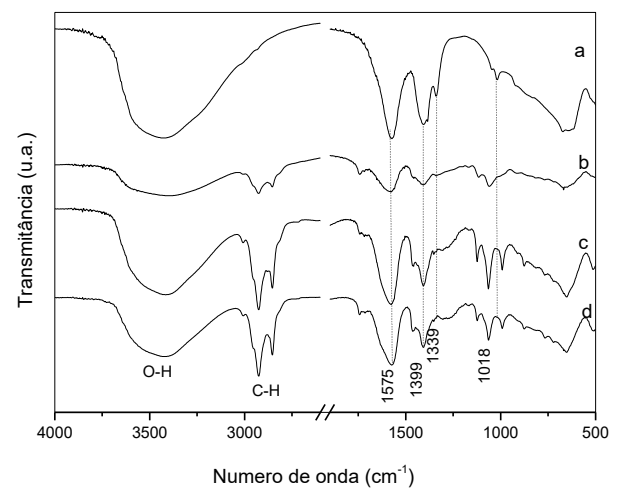

Observa-se que os espectros apresentam bandas em $3400 \mathrm{~cm}^{-1}$ associada a vibração de hidroxilas que fazem ligações de hidrogênio com a água. Em $1575 \mathrm{~cm}^{-1} \mathrm{e} \mathrm{em} 1399 \mathrm{~cm}^{-1}$, as bandas intensas surgem devido a presença de estiramentos assimétricos e simétricos dos grupos carboxilatos respectivamente. Em $1339 \mathrm{~cm}^{-1}$, a banda se refere à deformação simétrica do grupamento $-\mathrm{CH}_{3}$ e em $1018 \mathrm{~cm}^{-1}$ aparece a banda de rocking do grupamento metila (BISWICK et al., 2009). Sendo que estas bandas são preservadas após 3 ciclos de transesterificação metílica para produção de biodiesel, demonstrando que o material é majoritariamente preservado após três ciclos de reação. As bandas presentes na região abaixo de $1000 \mathrm{~cm}^{-1}$ se tornaram alargadas, devido à redução de cristalinidade do material e outras bandas são observadas na região de $800-1100 \mathrm{~cm}^{-1}$, atribuídas a uma pequena contaminação de material orgânico, provavelmente produtos residuais como ésteres e ácidos graxos. As bandas na região de $2800-2900 \mathrm{~cm}^{-1}$ são provavelmente relativas à contaminação do material com éster, o qual não foi totalmente eliminado por lavagem.

Os resultados da investigação de HAZN como catalisador na transesterificação do óleo de soja com metanol, portanto, provou-se satisfatório. Sendo que a conversão de ésteres para um uso e dois reusos foi semelhante, em torno de $70 \%$, o que combinado as demais análises demonstra que o catalisador sintetizado é também reutilizável, embora perca grande parte da cristalinidade original após cada reuso.

\section{REFERÊNCIAS}

ARIZAGA, G. G. C.; SATYANARAYANA, K. G.; WYPYCH, F.; Layered hydroxide salts: Synthesis, properties and potential applications. Solid State Ionics, 178, 1143-1162, 2007.

BISWICK, T.; JONES, W.; PACULA, A.; SERWICKA, E.; PODOBINSKI, J.; Evidence for the formation of anhydrous zinc acetate and acetic anhydride during the thermal 
degradation of zinc hydroxy acetate, $\mathrm{Zn} 5(\mathrm{OH}) 8(\mathrm{CH} 3 \mathrm{CO} 2) 2 \cdot 4 \mathrm{H} 2 \mathrm{O}$ to $\mathrm{ZnO}$. Solid State Sci., 11, 330-335, 2009.

BOEY, P.; MANIAM, G. P.; HAMID, S. A.; Performance of calcium oxideas a heterogêneous catalyst in biodiesel production: A review. Chemical Engineering Journal, v 168, p 15-22, 2011.

COELHO, A.; PERRONE, O. M,; GOMES, E. DA-SILVA, R.; THOMÉO, J.C.; BOSCOLO, M.; Mixed metal oxides from sucrose and cornstarch templated hydrotalcite-like LDHs as catalysts for ethyl biodiesel synthesis. Applied Catalysis A: General, v. 532 p. 32-39, 2017.

CORDEIRO, C. S.; SILVA, F. R.; WYPYCH, F.; RAMOS, L. P.; Catalisadores heterogêneos para a produção de monoésteres graxos (biodiesel). Quim. Nova, v 34, p 477-486, 2011.

KANDARE, E.; HOSSENLOPP, J. M.; Thermal Degradation of Acetate-Intercalated Hydroxy Double and Layered Hydroxy Salts. Inorg. Chem. v 45, p 3766-3773, 2006.

KOUZU, M.; KASUNO, T.; TAJIKA, M.; YAMANAKA, S.; HIDAKA, J.; Active phase of calcium oxide used as solid base catalyst for transesterification of soybean oil With refluxing methanol. Applied Catalysis A: General, v 334, p 357-365, 2008.

MARANGONI, R. Materiais Multifuncionais obtidos a partir da intercalação de corantes aniônicos em Hidróxidos Duplos e Hidroxissais Lamelares e Dispersos em Polímeros. Tese de doutorado, Dep. de Química, UFPR, 2009.

NANDIWALE, K. e BOKADE, V. Process Optimization by Response Surface Methodology and Kinetic Modeling for Synthesis of Methyl Oleate Biodiesel over $\mathrm{H}_{3} \mathrm{PW}_{12} \mathrm{O}_{4}$ Anchored Montmorillonite K10. Industrial and Engineering Chemistry. Research v. 53, p.18690-18698, 2014.

YAMANAKA, S.; ANDO, K.; OHASHI, M.; New Anion Exchangeable Layered Mixed. Basic Salt, $\mathrm{Ni}_{1-\mathrm{x}} \mathrm{Zn}_{2 \mathrm{x}}(\mathrm{OAc})_{2 \mathrm{x}}(\mathrm{OH})_{2} \cdot \mathrm{nH}_{2} \mathrm{O}$. Mater. Res. Soc. Symp. Proc. V 371, p 131142, 1995. 